\title{
ARTICLE OPEN \\ An efficient cell free enzyme-based total synthesis of a meningococcal vaccine candidate
}

\author{
Timm Fiebig ${ }^{1,3}$, Maria Rosaria Romano ${ }^{2,3}$, Davide Oldrini ${ }^{2}$, Roberto Adamo ${ }^{2}$, Marta Tontini ${ }^{2}$, Barbara Brogioni ${ }^{2}$, Laura Santini ${ }^{2}$, \\ Monika Berger ${ }^{1}$, Paolo Costantino ${ }^{2}$, Francesco Berti ${ }^{2}$ and Rita Gerardy-Schahn ${ }^{1}$
}

Invasive meningococcal disease (IMD) is a global health problem and vaccination has proven the most effective way of disease control. Neisseria meningitidis serogroup $\mathrm{X}(\mathrm{NmX})$ is an emerging threat in the African sub-Saharan meningitis belt, but no vaccine is available today. Leading vaccines against $\mathrm{Nm}$ are glycoconjugates, in which capsular polysaccharides isolated from large-scale pathogen cultures are conjugated to adjuvant proteins. Though safe and efficacious even in infants, high costs and biohazard associated with the production limit abundant application of glycoconjugate vaccines particularly in the most afflicted nations. An existing $N m X$ vaccine candidate (CPSXn-CRM ${ }_{197}$ ) produced by established protocols from NmX capsule polysaccharide (CPSX) has been shown to elicit high bactericidal immunoglobulin $\mathrm{G}$ titres in mice. Here we describe the scalable in vitro synthesis of CPSXiv from chemically pure precursors by the use of recombinant $\mathrm{NmX}$ capsule polymerase. Application of the described coupling chemistry gives CPSXiv-CRM 197 , which in mouse vaccination experiments behaves identical to the benchmark CPSXn-CRM $_{197}$. Excluding any biohazards, this novel process represents a paradigm shift in vaccine production and a premise towards vaccine manufacturing in emerging economies.

Npj Vaccines (2016) 1, 16017; doi:10.1038/npjvaccines.2016.17; published online 15 November 2016

\section{INTRODUCTION}

Neisseria meningitidis (Nm) is a leading cause of bacterial meningitis and sepsis worldwide. The strictly human pathogen causes recurrent devastating epidemics in developing countries, in particular the African sub-Saharan meningitis belt. ${ }^{1}$ In Western World countries, $\mathrm{Nm}$ infections occur sporadically, but outbreaks have been reported caused by hyper-invasive strains in situations of crowding ${ }^{2,3}$ and in communities with increased risk. ${ }^{4,5}$ As Nm is the only bacterial pathogen that can spread in epidemic waves, outbreaks present a significant danger for individuals with high susceptibility, that is, small children, adolescents and elderly people. ${ }^{6}$

A chief problem with invasive meningococcal disease (IMD), often characterised by meningitis and sepsis, is rapid progression. IMD can lead to death within hours, ${ }^{7}$ leaving open an extremely short window for medical intervention. Accordingly, case-fatality rates are high, exceeding $20 \%$ even in developed countries. ${ }^{8}$ Moreover, survivors often suffer from fatal sequelae like limb loss, deafness and neurologic disabilities. ${ }^{9}$ The benefit of vaccination is obvious on this background and motivated industrial companies (GSK Vaccines, Pfizer, Baxter, Sanofi-Pasteur, Serum Institute of India ${ }^{10}$ ) and international nonprofit organisations like PATH and $\mathrm{WHO}^{11,12}$ to invest into the development of vaccines to combat IMD and thus improve health and life conditions in afflicted regions.

Major virulence factors of $\mathrm{Nm}$ are the capsular polysaccharides (CPS). ${ }^{13}$ Twelve chemically different CPSs have been identified and determine the twelve $\mathrm{Nm}$ serogroups, of which six $(\mathrm{NmA},-\mathrm{B},-\mathrm{C}$, $-W,-Y$ and $-X)$ account for virtually all cases of IMD. ${ }^{13}$ With the exception of NmB, where the CPS (CPSB) consists of a2,8-linked polysialic acid identical to host-expressed polysialic acid, ${ }^{14,15}$ the CPSs coupled to adjuvant proteins provide the basis for modern vaccine formulations, the so called glycoconjugate vaccines. ${ }^{16}$ Currently, mono- and multivalent glycoconjugate vaccines are available for $\mathrm{NmA},-\mathrm{C},-\mathrm{W}$ and $-\mathrm{Y}$ and their application has provided most promising results. ${ }^{16}$

Though safe and effective, glycoconjugate vaccines are under debate because of high production costs that may limit broad application in afflicted regions. ${ }^{17}$ Up until today, the production starts with mass fermentation of pathogens, a biohazardous step that requests the high-tech infrastructure of modern industrial plants ${ }^{10}$ and limits the build-up of the production infrastructure in indigent regions like the countries of the African sub-Saharan meningitis belt. However, the exigence of decentralised vaccine production has gained conspicuous actuality in the 2015 meningitis season, when an NmC outbreak in Niger could not be stopped because of a shortage in vaccine provision. ${ }^{18}$

Considering that no licenced $N m \mathrm{X}$ vaccine exists today and capitalising on our recent success with exploiting recombinant capsule polymerases (CPs) for in vitro production of bioidentical CPSs, ${ }^{19,20}$ we focused this study on the production of a synthetic $N m X$ vaccine. With recombinant CsxA, the CP of $N m X$, we established reaction conditions that yielded the nature identical in vitro produced CPSX (henceforth referred to as CPSXiv to differentiate it from CPSXn, the polymer isolated from natural source) in homogenous quality. In a mouse vaccination experiment, a glycoconjugate vaccine prepared from CPSXiv was found to induce bactericidal antibodies at titres not different form the benchmark. ${ }^{21}$ The process line exemplified for $N m \mathrm{X}$ can be easily adjusted for the production of vaccines against other $\mathrm{Nm}$

\footnotetext{
${ }^{1}$ Institute for Cellular Chemistry, Hannover Medical School, Hannover, Germany and ${ }^{2}$ GSK Vaccines, Research, Siena, Italy.

Correspondence: F Berti or R Gerardy-Schahn (francesco.x.berti@gsk.com or gerardy-schahn.rita@mh-hannover.de)

${ }^{3}$ These authors contributed equally to this work.

Received 10 May 2016; revised 14 July 2016; accepted 3 August 2016
} 
serogroups and thus should have model character for all glycoconjugate vaccines that start with the biohazardous step of pathogen fermentation.

\section{RESULTS}

In vitro synthesis of oligosaccharide primers

Recently we described the small scale production of CPSXiv by use of recombinant CsxA. ${ }^{19}$ Besides the chemically pure donor sugar UDP-N-acetylglucosamine (UDP-GICNAC), short priming oligosaccharides (primCPSX) were needed to maximise the reaction efficacy and were obtained through hydrolysis of CPSXn. ${ }^{19}$ As it was our aim to avoid the use of Neisseria-derived materials to the greatest possible extent in the current upscaled reactions, we first produced primCPSXiv (Figure 1a). Still starting with primCPSXn, three consecutive CsxA catalysed reactions (reactions 1-3) were carried out. Priming oligosaccharides in reactions 2 and 3 were derived from purified and hydrolysed CPSXiv produced in reactions 1 and 2 , respectively. In reactions $1-3$, priming oligosaccharides were mixed with a 1,000-fold molar excess of UDP-GICNAc, resulting in a $>10^{9}$ fold dilution of the initially used primCPSXn. Because acidic hydrolysis cleaves the phosphodiester bond in CPSX proximal to the anomeric C-atom (Figure 1b), oligosaccharides obtained from reaction 3 were enzymatically treated to release the phosphate group from the non-reducing end and thus maximise the concentration of functional primers. Anion-exchange chromatography (AEC) used to purify this final primCPSXiv pool as well as high-performance anion-exchange chromatography with pulsed amperometric detection (HPAEC$P A D$; Figure 1a) identified fragments ranging in size between
DP1-DP8 (DP, degree of polymerisation), for which an average DP (avDP) of 4.5 was calculated based on ${ }^{1} \mathrm{H}$ NMR analysis. ${ }^{21}$

Determination of efficient reaction conditions

Because the donor-substrate UDP-GICNAc (henceforth referred to as donor, D) is the single reaction component of appreciable economic value, completeness of conversion into CPSXiv is a critical aspect. With the primCPSXiv fraction of avDP4.5 (henceforth referred to as acceptor, A) at hand, a titration experiment was carried out to determine the D/A ratio that allowed complete consumption of UDP-GICNAc. The donor concentration was kept at $10 \mathrm{mM}$ and the acceptor increased to give D/A ratios between 4,600 and 90 (Figure 1c). The reactions were run overnight and products separated and visualised using our recently developed high-performance liquid chromatography based anion exchange chromatography (HPLC-AEC) assay. ${ }^{20}$ Complete conversion of UDP-GICNAC to UMP was seen at a D/A ratio of 460 . However, because the basis of the product peak, an indicator of product dispersity, had considerably broadened from D/A ratio of 950 to D/A ratio of 460 , we decided for the D/A ratio of 800 to set-up medium-scale CPSXiv productions.

\section{Scaling up CPSXiv production and product characterisation}

The first upscaled reaction was calculated to give $35 \mathrm{mg}$ CPSXiv. As before, UDP-GICNAc consumption and product synthesis were visualised by HPLC-AEC (shown for start and end point in Figure 2a). After overnight incubation all UDP-GlcNAc was converted to UMP with the corresponding CPSXiv peak detected at $214 \mathrm{~nm}$. A close to $100 \%$ reaction yield was confirmed when the
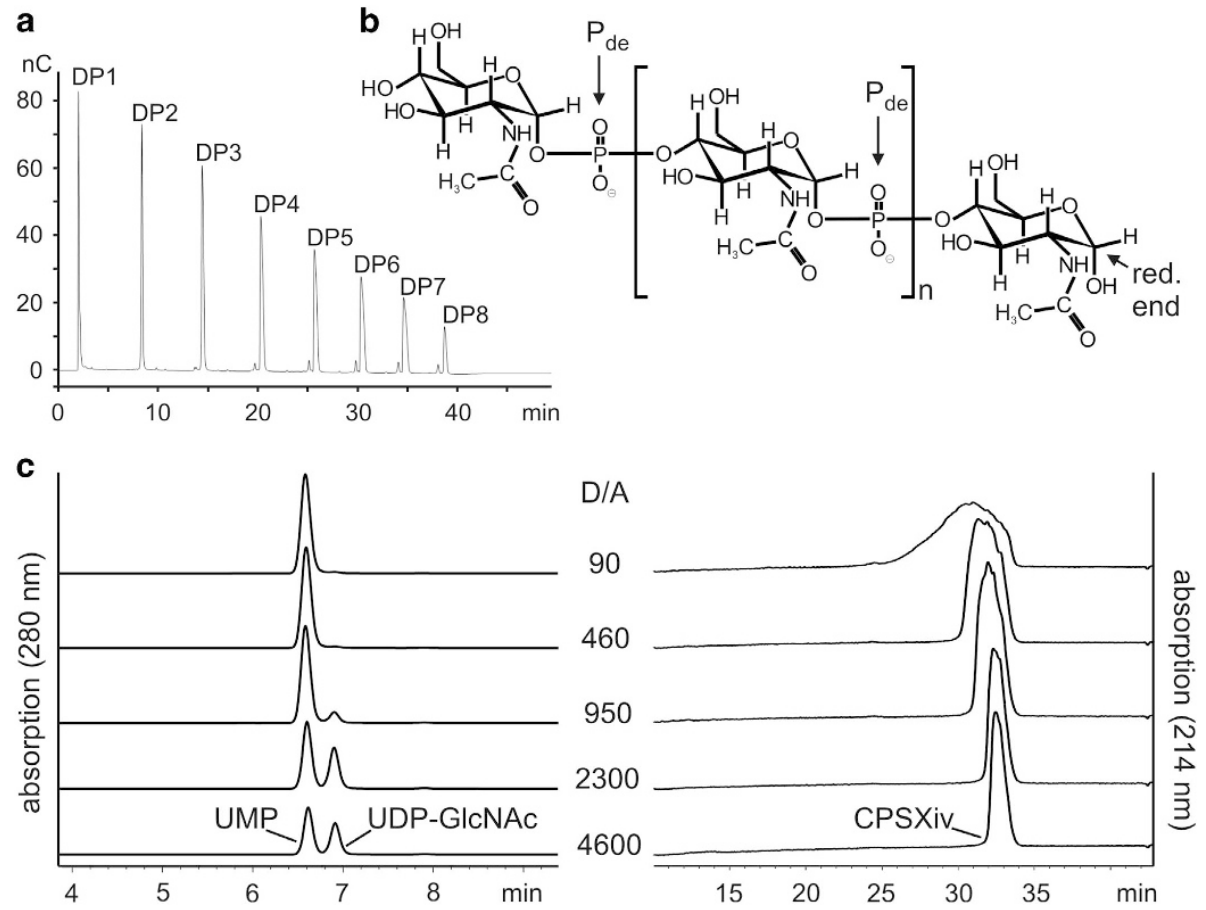

Figure 1. The priming oligosaccharide fraction and evaluation of reaction conditions. (a) HPAEC-PAD profiling shows that the primCPSXiv pool used for upscaling the CPSXiv synthesis contains the (oligo)saccharide species DP1-DP8. The minor peaks preceeding DP3-DP8 indicate the presence of small amounts of oligomers with non-reducing end phosphate groups. (b) Chemical structure of CPSX. Pde, phosphodiester; red. end, reducing end. (c) To determine the D/A ratio that allows complete consumption of UDP-GlcNAc, CsxA reactions were carried out with a constant donor (10 mM UDP-GlcNAc) and varying acceptor (primCPSXiv) concentrations to give D/A ratios as indicated. After overnight incubation, the product spectra were analysed by HPLC-AEC. Although a small peak indicating residual UDP-GlcNAc was visible at the D/A ratio 950, conversion to UMP was complete at D/A ratio 460. CPSX, NmX capsule polysaccharide; CPSXiv, in vitro produced CPSX; HPAEC-PAD, high-performance anion-exchange chromatography with pulsed amperometric detection; HPLC-AEC, high-performance liquid chromatography based anion exchange chromatography. 

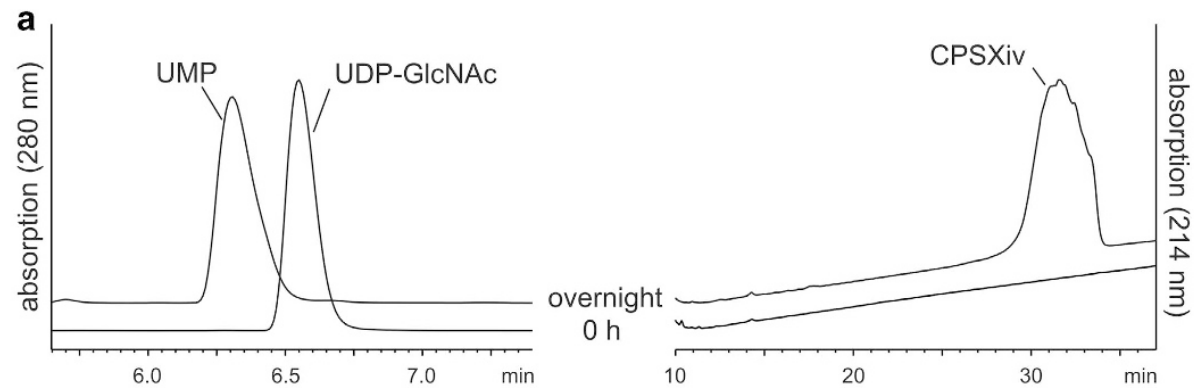

b

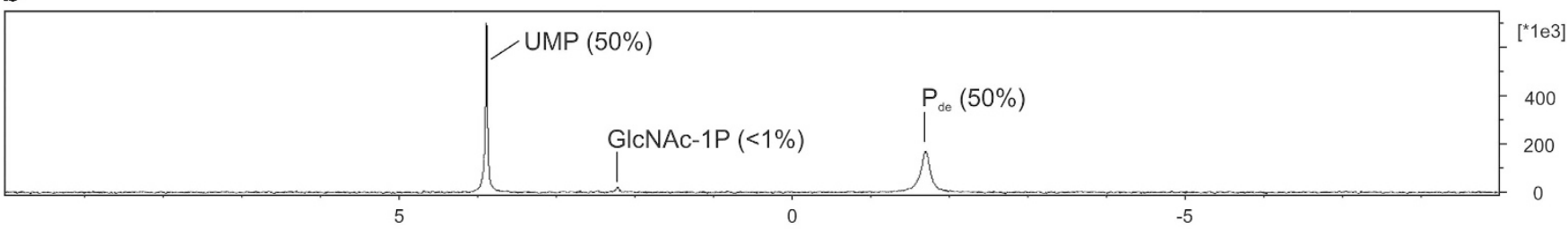

C

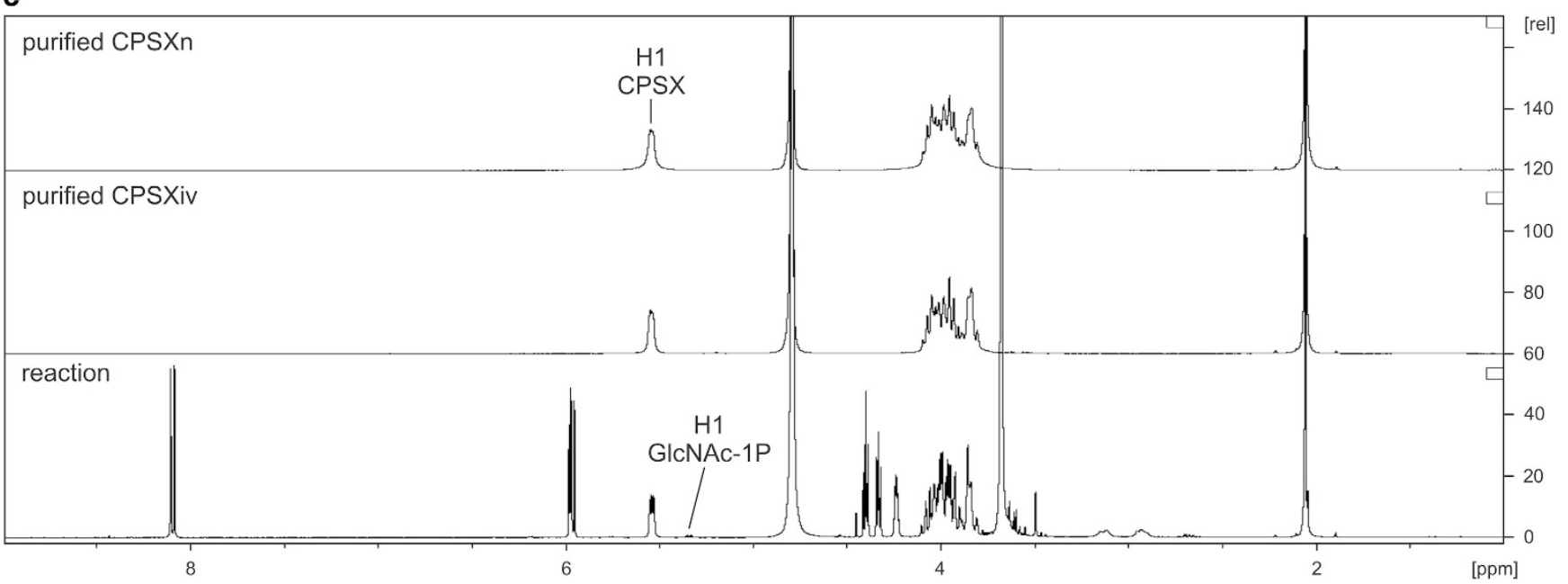

Figure 2. Synthesis and physico-chemical characterisation of CPSXiv. (a) Completeness of the reaction scaled to give 35 mg CPSXiv was monitored by HPLC-AEC. After overnight incubation, the donor (UDP-GlcNAC) peak was replaced by a UMP peak and the corresponding CPSXiv peak was recorded at $214 \mathrm{~nm}$. (b) ${ }^{31}$ P NMR analysis of the reaction mixture identified two major peaks representing UMP and $P_{\text {de. }}$ Peak area integration gave equal numbers, thus confirming the quantitative integration of the donor into CPSXiv. The signal for the side product GIcNAc-1P did not exceed trace concentration. (c) ${ }^{1} \mathrm{H}$ NMR used to control purification results and chemical identity between CPSXiv and CPSXn confirmed both, homogeneity and bio-identity of the CPSXiv fraction. CPSX, NmX capsule polysaccharide; CPSXiv, in vitro produced CPSX; CPSXn, CPSX obtained from natural source; HPLC-AEC, high-performance liquid chromatography based anion exchange chromatography; p.p.m., parts per million.

mixture was analysed by ${ }^{31} \mathrm{P}$ NMR (Figure $2 \mathrm{~b}$ ). Two main peaks representing UMP and $\mathrm{P}_{\mathrm{de}}$ (phosphodiester bonds) appeared and integration of peak areas resulted in a 1:1 ratio. With only trace amounts of GlcNAc-1P, a product of a hydrolytic side activity of $\mathrm{CsxA}^{19}$ the highly sensitive ${ }^{31} \mathrm{P}$ NMR analysis corroborated the chosen reaction conditions. To control reproducibility, a second reaction was calculated to give $112 \mathrm{mg}$ and was carried out in triplicate. The analytical HPLC-AEC profiles obtained for these reactions clearly confirmed the reliability of the CPSXiv production procedure (Supplementary Figure 1).

Because the CPSXiv production was started with ultrapure reagents and yielded close to $100 \%$ donor consumption, the product purification steps were limited to salt removal by dialysis and AEC to separate CPSXiv from UMP and GlcNAc-1P traces (data not shown). Product purity and identity were confirmed by ${ }^{1} \mathrm{H}$ NMR (Figure 2c). If compared with the crude reaction mixture (bottom panel), it is immediately obvious that the two-step purification procedure delivered homogenously pure CPSXiv (middle panel). Moreover, co-analysis of CPSXn (top panel) demonstrated chemical identity of the two polymers. As a final step, we compared the size of CPSXiv and CPSXn chains using HPLC size exclusion chromatography. With a relative molecular mass of $308 \mathrm{kDa}$, CPSXiv perfectly mimicked the natural product CPSXn with a relative molecular mass of $388 \mathrm{kDa}$ (Supplementary Figure 2a).

Preparation of a CPSXiv-based glycoconjugate vaccine For the preparation of a CPSXiv-based vaccine we precisely followed the protocol previously established for CPSXn. ${ }^{21}$ First, $120 \mathrm{mg}$ of the synthetic polymer were hydrolysed to shorten the CPS chains and the reaction was monitored by ${ }^{31} \mathrm{P}$ NMR. ${ }^{22}$ Hydrolysis was stopped after $270 \mathrm{~min}$ when ${ }^{31} \mathrm{P}$ NMR analysis roughly indicated an avDP12 (Supplementary Figure 2b). The mixture was loaded onto an AEC column to remove oligosaccharides shorter than five to six repeating units. Two pools containing 
a

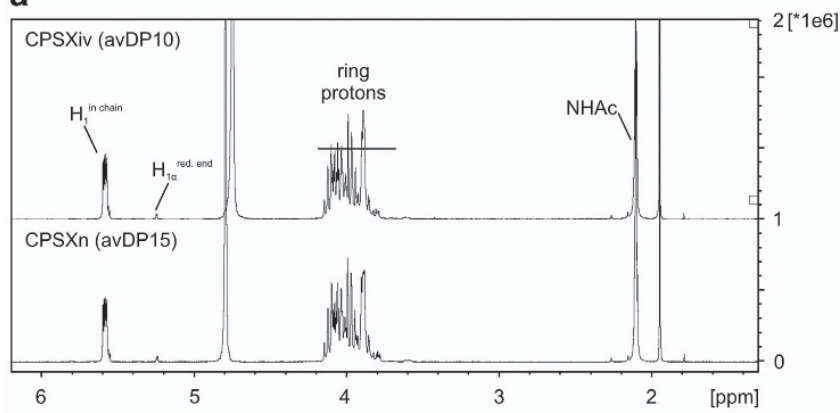

b

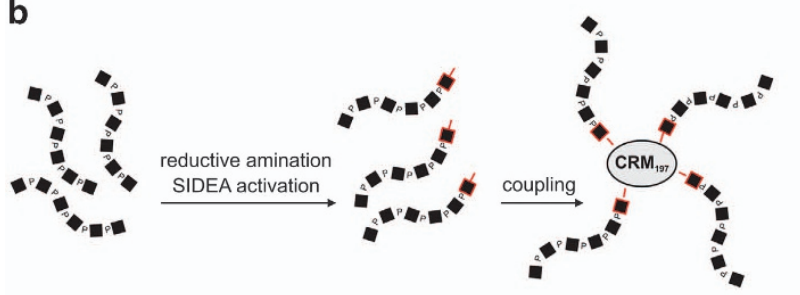

C

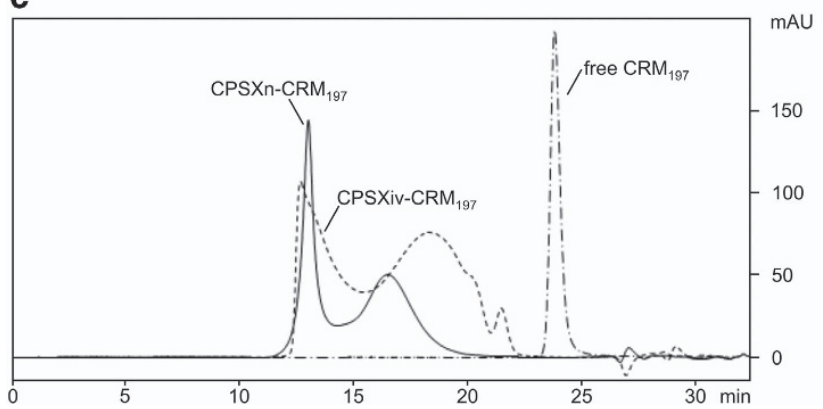

Figure 3. Generation of a CPSXiv-based glycoconjugate. (a) To ensure matching of the oligosaccharide fraction generated from CPSXiv and CPSXn, ${ }^{1} \mathrm{H}$ NMR spectra were recorded and showed perfect correspondence. (b) Schematic representation of CPSX hydrolysis and coupling of the resulting oligosaccharides to the protein carrier $\mathrm{CRM}_{197}$. The reducing end sugars are marked in red. (c) The efficacy of oligosaccharide coupling to $\mathrm{CRM}_{197}$ was controlled by HPLC size exclusion chromatography with detection of the conjugates via absorption at $214 \mathrm{~nm}$. Similar to the benchmark $\left(C P S X n-C_{197}\right), C_{1}$ CPSXiv-CRM ${ }_{197}$ elutes in two major peaks. In line with the lower avDP of CPSXiv oligosaccharides (avDP10, Pool 2), the second peak showed a mild shift to lower molecular masses. Importantly, no free $\mathrm{CRM}_{197}$ was detected. CPSX, NmX capsule polysaccharide; CPSXiv, in vitro produced CPSX; CPSXn, CPSX obtained from natural source; HPLC, high-performance liquid chromatography; p.p.m., parts per million.

different oligosaccharide polydispersions were collected (Supplementary Figure 2c). Pool 2 contained $84.7 \mathrm{mg}$ of oligosaccharides (70\% recovery), which, as identified in an analytical HPAEC-PAD run, exhibited an overlapping dispersity with the benchmark CPSXn of avDP15 (Supplementary Figure 2d). However, with avDP10 (calculated based on ${ }^{31} \mathrm{P}$ NMR analysis; see Supplementary Figure 1d), pool 2 slightly deviated from the benchmark (avDP15). Finally, the perfect congruence of the ${ }^{1} \mathrm{H}$ NMR spectra obtained for the oligosaccharide fractions prepared from CPSXiv and CPSXn confirmed their chemical identity (Figure 3a).

Conjugation of CPSXiv fragments to the protein carrier $\mathrm{CRM}_{197}$, a non-toxic variant of the diphtheria toxin, ${ }^{23}$ followed the coupling technology successfully used by Micoli et $a l^{21}$ to produce the CPSXn-based vaccine MenX-ADH-SIDEA-CRM 197 (referred to as
Table 1. Comparative characterisation of CPSXiv-CRM 197 and $\mathrm{CPSXn}^{-\mathrm{CRM}_{197}}$

\begin{tabular}{|c|c|c|c|}
\hline & $\begin{array}{l}\text { Saccharide/ } \\
\text { protein } \\
\text { ratio }(w / W)\end{array}$ & $\begin{array}{c}\text { Saccharide/ } \\
\text { protein } \\
\text { ratio }(\mathrm{mol} / \mathrm{mol})\end{array}$ & $\begin{array}{l}\text { Unconjugated } \\
\text { saccharide \% }\end{array}$ \\
\hline CPSXiv-CRM $_{197}$ & 0.2 & 4.1 & $<6.0$ \\
\hline CPSXn-CRM $_{197}$ & 0.3 & 4.1 & $<3.5$ \\
\hline \multicolumn{4}{|c|}{$\begin{array}{l}\text { Abbreviations: CPSX, NmX capsule polysaccharide; CPSXiv, in vitro pro } \\
\text { duced CPSX; CPSXn, CPSX obtained from natural source. } \\
\text { The saccharide/protein ratios (mol/mol) for CPSXiv-CRM }{ }_{197} \text { anc } \\
\text { CPSXn-CRM } 197 \text { were calculated using the average molecular weigh } \\
\text { of the respective fraction (avDP10 for CPSXiv-CRM }{ }_{197} \text { and avDP15 fo } \\
\text { CPSXn-CRM } 197 \text { ). }\end{array}$} \\
\hline
\end{tabular}

CPSXn-CRM 197$).{ }^{21}$ As schematically illustrated in (Figure 3b), coupling to $\mathrm{CRM}_{197}$ proceeds via the reducing end of the oligosaccharides (marked by red squares). Successful coupling was confirmed by HPLC size exclusion chromatography. Similar to our benchmark, CPSXn-CRM 197 , the new glycoconjugate CPSXiv$\mathrm{CRM}_{197}$ showed a bipartite elution profile in the absence of detectable amounts of free $\mathrm{CRM}_{197}$ (Figure 3c). However, in line with the smaller size of the oligomer fraction, the second peak was shifted right. CPSXiv-CRM 197 was then purified by hydrophobic interaction chromatography and, as a final step in the quality control process, established protocols ${ }^{21,24}$ were used to determine oligosaccharide loading of $\mathrm{CRM}_{197}$ and control for the presence of free saccharide. These control experiments were done in parallel with CPSXn-CRM $_{197}$ (Table 1).

\section{Immunogenicity testing in mice}

The ability of CPSXiv-CRM 197 to elicit protective antibodies was tested in a mouse model in comparison with CPSXn-CRM ${ }_{197 .}{ }^{21}$ Figure 4 a illustrates the immunisation scheme. After the collection of pre-immune sera at day 0 , female BALB/c mice (eight animals per group) were subcutaneously injected with the first dose (day $1 ; 1 \mu \mathrm{g}$ saccharide content per dose, formulated in phosphatebuffered saline buffer, $\mathrm{pH} 7.2$, with aluminum phosphate as adjuvant). The injections were repeated twice at day 14 and day 28. Post immunisation, sera (post) were taken at day 27 (Post 2) and day 42 (Post 3). Control animals were treated in the same scheme with phosphate-buffered saline plus adjuvant. Immunoglobulin $\mathrm{G}(\mathrm{IgG})$ titres induced were detected using our recently developed ELISA (enzyme-linked immunosorbent assay). ${ }^{21}$ Comparable IgG titres obtained with the two glycoconjugate vaccines indicate identical immunological properties for CPSXiv-CRM 197 and CPSXn-CRM 197 (Figure 4b). Moreover, it is of note that considerable IgG titres had established already after two injections (Post 2 sera) and only increased by factor 2-3 after the third vaccine application (CPSXn-CRM ${ }_{197}: P=0.028, \quad$ CPSXiv-CRM $_{197}$ : $P=0.0284$; Mann-Whitney test).

Eventually we measured bactericidal activity against $\mathrm{NmX}$ strain Z9615. Here, sera isolated from each group and time point were pooled and rabbit serum was used as complement source. ${ }^{21}$ Comparable bactericidal activity (one dilution step is within the variation of the assay) measured in sera collected from CPSXiv$\mathrm{CRM}_{197}$ and $\mathrm{CPSXn}_{\mathrm{C}} \mathrm{CRM}_{197}$ immunised mice convincingly evidenced the value of the described alternative and biohazard-free protocol for the production of an $\mathrm{NmX}$ vaccine (Table 2).

\section{DISCUSSION}

For the first time, we describe in this study the use of in vitro synthesised nature identical CPSXiv for the production of a 
a

\begin{tabular}{|c|c|c|c|}
\hline Day $0 / 1$ & Day 14 & Day $27 / 28$ & Day 42 \\
\hline $\begin{array}{l}\text { preimmune serum / } \\
\text { 1. dose }\end{array}$ & 2. dose & $\begin{array}{l}\text { Serum post } 21 \\
\text { 3. dose }\end{array}$ & post 3 \\
\hline
\end{tabular}

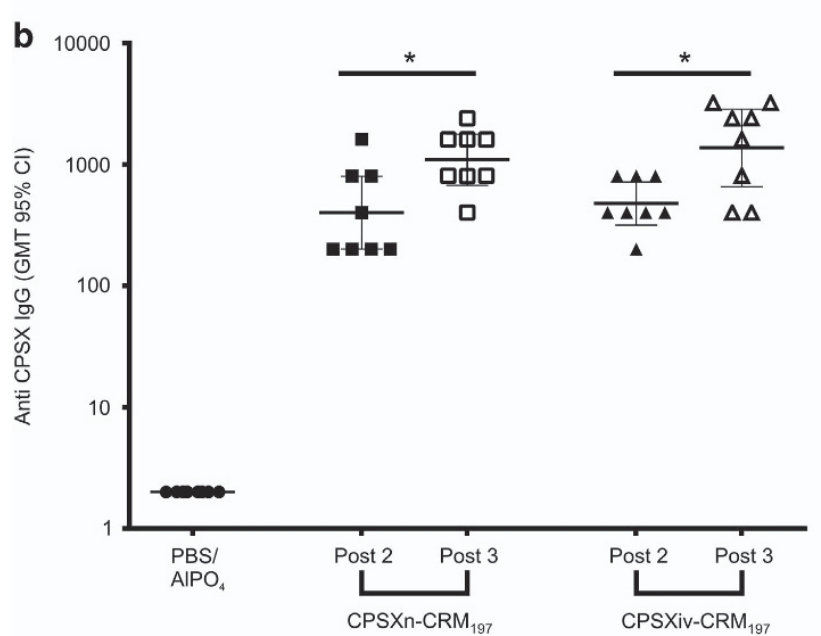

Figure 4. Immunogenicity of CPSXiv-CRM $\mathrm{CR}_{197}$ in comparison with CPSXn-CRM 197 . (a) Vaccination schedule showing the immunisation and bleeding of mice. (b) The anti-CPSX IgG ELISA used to determine immunisation results demonstrated comparable IgG responses to CPSX with both, the synthetic (CPSXiv-CRM ${ }_{197}$ ) and the benchmark (CPSXn-CRM ${ }_{197}$ ) vaccine. Each symbol represents an individual animal. The horizontal bars indicate the geometric mean titre and $95 \%$ confidence intervals. *Significant differences between the indicated groups with $P<0.05$ (CPSXn-CRM ${ }^{197}: P=0.028$, CPSXiv-CRM $^{197}: P=0.0284$; Mann-Whitney test). CPSX, NmX capsule polysaccharide; CPSXiv, in vitro produced CPSX; CPSXn, CPSX obtained from natural source; IgG, immunoglobulin G.

Table 2. Rabbit serum bactericidal activity assay

\begin{tabular}{lcc}
\hline Group & Post 2 (pooled sera) & Post 3 (pooled sera) \\
\hline PBS/AIPO $_{4}$ & $<4$ & $<4$ \\
CPSXn-CRM $_{197}$ & 2,048 & 8,192 \\
CPSXiv-CRM $_{197}$ & 1,024 & 4,096 \\
\hline
\end{tabular}

Abbreviations: CPSX, NmX capsule polysaccharide; CPSXiv, in vitro produced CPSX; CPSXn, CPSX obtained from natural source; PBS, phosphatebuffered saline.

Post 2 and Post 3 sera were pooled for the respective groups of mice. Bactericidal titres are defined as the reciprocal serum dilution that gives a $50 \%$ decrease of colony-forming units after $60 \mathrm{~min}$ incubation in the reaction mixture, compared with the mean number of colony-forming units in the control reactions at time point zero.

synthetic NmX glycoconjugate vaccine candidate (CPSXiv-CRM ${ }_{197}$ ). In a mouse immunisation study, CPSXiv-CRM ${ }_{197}$ competed with the benchmark vaccine CPSXn-CRM 197 (ref. 21) in terms of immunogenicity and elicitation of bactericidal antibodies. We chose $\mathrm{NmX}$ to pioneer the enzyme-catalysed vaccine production process, because this serogroup has gained prevalence worldwide ${ }^{17,25-31}$ and outbreaks in Niger, ${ }^{25}$ Uganda and Kenya, ${ }^{31}$ and in Togo and Burkina Faso ${ }^{17,30}$ have forcefully evidenced the need for a specific vaccine. Moreover, in the absence of a licensed $\mathrm{NmX}$ vaccine, the implementation of an alternative production scheme should be expedited.

As highlighted by the Global Alliance for Vaccines and Immunisation, a key determinant towards improved health and economic conditions in low-income countries is their access to vaccines (vaccines against poverty). ${ }^{32-34}$ The production process described in this study should be a quantum leap in this regard. Starting from well-defined and pyrogen-free chemicals (UDP-GlcNAc and primCPSXiv) and completely excluding biohazards, the process provides a vantage ground for the build-up of the production infrastructure in indigent regions and thus increase their flexibility to reply to unforeseen needs. ${ }^{18}$ In addition, stockpiling of vaccines and of vaccine components (for example, oligosaccharide fractions ready for coupling) as requested by the International Coordinating Group on Vaccine Provision for Epidemic Meningitis would be facilitated with the new technology. Of utmost importance in this context is that analytical steps to guarantee safety, homogeneity and lot consistency are facilitated in the fermentation-free procedure. ${ }^{35}$

The described conditions allowed close to $100 \%$ conversion of the donor sugar into polymer. Small oligosaccharides that accrue in hydrolysis and sizing steps provide the optimal starters for new production rounds and a simple HPLC-based analysis enabled online process control. Last, but certainly not the least, the recombinant CsXA can be produced in high quality. Without any further optimisation, the enzyme purified from only 1 litre of nonpathogenic E. coli M15(pREP4) culture $\left(\mathrm{OD}_{600}=3.0\right.$; ref. 19) would have sufficed to generate $8 \mathrm{~g}$ CPSXiv. This value is remarkable, given the fact that yields of homogenously pure CPSXn (isolated from fermented bacteria) range between 10 and $600 \mathrm{mg} / \mathrm{ll}^{21,36-39}$ Nevertheless, significant room remains for further process optimisation, in particular at the level of the recombinant enzyme. First, solid phase coupling and reuse of the enzyme in repeated production rounds would simplify and enhance the process. Second, the capsule polymerase per se can be engineered towards enhanced yield, solubility and/or stability, ${ }^{20,40}$ to acquire processadjusted elongation modes, ${ }^{41}$ and, eventually, increased flexibility towards chemically functionalised acceptors. The latter could help reducing the coupling chemistry. ${ }^{20}$

A difference detected between the tested vaccines was a variation in the oligosaccharide dispersion (avDP10 in CPSXiv$\mathrm{CRM}_{197}$ versus avDP15 in CPSXn-CRM ${ }_{197}$ ), a feature most likely attributable to the smaller scale of the CPSXiv hydrolysis reaction. Although more studies are needed to finally decide if this difference is of relevance, the fact that immunisation results did not reflect any associated variance suggests that it is negligible.

In contrast to CPSX, which represents a simple homopolymer of GlcNAc-1P repeating units, CPSs from other $\mathrm{Nm}$ serogroups are more complex and in vitro production would need more than one enzymatic activity. We could recently demonstrate on an analytical scale that the herein-presented technology can potentially be expanded to more complex biosynthesis systems. The acetylated CPS of NmA could be produced in a one-pot reaction using three different recombinant enzymes, ${ }^{20}$ we have done pioneering steps towards the fermentation-free production of the dimeric CPSs of $N m W$ and $\mathrm{Y}^{42,43}$ and, as an example for the most complex CPS expressed by $\mathrm{Nm}$, we also described the synthesis of the trimeric CPS of $\mathrm{NmL}^{44}$

Taken together, this paradigmatic evaluation of a biohazardfree protocol for the production of an efficient $N m X$ vaccine should pave the way for future decentralised glycoconjugate vaccine production and thus back up the mission for vaccines against poverty. ${ }^{32}$

\section{MATERIALS AND METHODS}

Synthesis and purification of primCPSXiv

CPSXiv was synthesised in vitro using the recombinant capsule polymerase CsxA expressed as MBP-CsxA-His 6 fusion construct. ${ }^{19}$ Hydrolysis of $0.5 \mathrm{mg}$ CPSXiv $(2.5 \mathrm{mg} / \mathrm{ml})$ was performed in $50 \mathrm{mM}$ sodium acetate buffer at $\mathrm{pH}$ 4.0 and $80^{\circ} \mathrm{C}$ for $6 \mathrm{~h}$. NaOH was added to readjust $\mathrm{pH} 7$ before the mixture was dialysed against water (ZelluTrans, Roth, $1 \mathrm{kDa}$ MWCO) and freeze-dried. Alternatively, primCPSXiv were recycled from pool 1 containing oligosaccharides <DP10 (see Supplementary Figure 1c). For the 
dephosphorylation of oligosaccharides, we used either acid phosphatase (Worthington Biochemical Corporation, Lakewood, NJ, USA) or calf intestinal alkaline phosphatase (CIP, NEB) and followed the manufacturer's guidelines. Although removal of acid phosphatase from primCPSXiv was achieved with Amicon centrifugal devices (10 MWCO), CIP was removed by AEC. Individual oligosaccharides were separated on an ÄKTA-FPLC (GE Healthcare, Little Chalfont, Buckinghamshire, UK) equipped with a MonoQ HR $5 / 5$ column (GE Healthcare) at a flow-rate of $1 \mathrm{ml} / \mathrm{min} . \mathrm{H}_{2} \mathrm{O}$ and $1 \mathrm{M}$ $\mathrm{NaCl}$ were used as mobile phases $\mathrm{M}_{1}$ and $\mathrm{M}_{2}$, respectively. The samples were separated using a combination of linear gradients $\left(0\right.$ to $5 \% M_{2}$ over $1 \mathrm{ml}, 5$ to $20 \% \mathrm{M}_{2}$ over $10 \mathrm{ml}, 20$ to $30 \% \mathrm{M}_{2}$ over $20 \mathrm{ml}$ ). Saccharidecontaining fractions were pooled and desalted by dialysis (ZelluTrans, Roth, $1 \mathrm{kDa}$ MWCO) against a 1,000-fold excess of water. Fractions containing DP3, DP4 and DP5 were isolated and the DP was confirmed by ${ }^{1} \mathrm{H}$ NMR and HPAEC-PAD as described in refs 21,22 .

\section{Upscaling of the in vitro CPSX synthesis}

Test reactions in $25 \mu \mathrm{l}$ of total reaction volume were performed as described before ${ }^{19}$ in the presence of varying amounts of primCPSXiv to determine conditions suitable for the production of long CPSX chains and complete consumption of UDP-GICNAc. The analysis of the reaction products by HPLC-AEC was performed following established protocols. ${ }^{19,20,44}$ For upscaling, $80 \mathrm{mg}$ of UDP-GICNAC were incubated with 0.6 or $2.5 \mathrm{nmol}(62.5$ or $250 \mu \mathrm{g}$, for overnight or $4 \mathrm{~h}$ reactions, respectively) MBP-CsxA-His 6 at $37^{\circ} \mathrm{C}$ in the presence of primCPSXiv in a total volume of $12.4 \mathrm{ml}$ allowing the synthesis of a maximum of $35 \mathrm{mg}$ CPSXiv. The synthesis of $112 \mathrm{mg}$ CPSX was upscaled accordingly. UMP and other reaction constituents were removed by Tangential Flow Filtration using Vivaflow 50 membranes with $30 \mathrm{kDa}$ MWCO. Alternatively, the reaction volume was reduced to $5 \mathrm{ml}$ by freeze-drying and applied to an ÄKTA-FPLC (GE Healthcare) equipped with a MonoQ 10/100 GL column (GE Healthcare) at a flow-rate of $5 \mathrm{ml} / \mathrm{min} . \mathrm{H}_{2} \mathrm{O}$ and $1 \mathrm{M} \mathrm{NaCl}$ were used as mobile phases $M_{1}$ and $M_{2}$, respectively. The separation was performed using a combination of linear gradients from 0 to $15 \% \mathrm{M}_{2}$ over $24 \mathrm{ml}$ and from 15 to $100 \% \mathrm{M}_{2}$ over $224 \mathrm{ml}$. CPSX-containing fractions were pooled, dialysed against $\mathrm{H}_{2} \mathrm{O}$ and freeze-dried. Acidic hydrolysis was performed for $4.5 \mathrm{~h}$ as described above and the avDP was estimated by ${ }^{31} \mathrm{P}$ NMR. ${ }^{22}$ The hydrolysate was loaded onto a Q Sepharose column (GE Healthcare) equilibrated with binding buffer ( $5 \mathrm{mM}$ sodium acetate, $\mathrm{pH}$ 7.0). Small oligosaccharides ( $<$ DP5-6) were removed with binding buffer containing $200 \mathrm{mM} \mathrm{NaCl}$ (pool l; Supplementary Figure 1c) and longer oligosaccharides were eluted with binding buffer containing $1 \mathrm{M} \mathrm{NaCl}$.

\section{Generation and immunogenicity of CPSXn-CRM 197}

Immunisation protocol, serum analysis, ELISA and rSBA. All methods concerning the generation and characterisation of CPSXiv-CRM 197 as well as the immunisation protocol and serum analysis were performed as described before. ${ }^{21} \mathrm{NmX}$ strain Z9615 was kindly provided by Gerd Pluschke, Swiss Tropical and Public Health Institute, Molecular Immunology, Basel, Switzerland. The animal studies were performed in accordance with the Italian law, approved by the local Animal Ethics Committee, and authorised by the Italian Ministry of Health.

\section{ACKNOWLEDGEMENTS}

We thank Daniela Proietti, Evita Balducci and Elena Mori of GSK Vaccines (Siena, Italy) for useful discussions and Gerd Pluschke of Swiss Tropical and Public Health Institute, Molecular Immunology (Basel, Switzerland), for kindly providing NmX strain Z9615. The study was financed by LOM (impact oriented funding) funds to the Institute for Cellular Chemistry.

\section{CONTRIBUTIONS}

RG-S and PC initiated the research; TF, RG-S, FB and MRR designed the research; TF, $M R R, D O, M T, B B, L S$ and MB performed the research; RG-S, FB, TF, RA and MRR interpreted the results; TF and RG-S supported by FB, MRR, RA and PC wrote the manuscript.

\section{COMPETING INTERESTS}

The authors MRR, DO, RA, MT, BB, LS, PC and FB are full-time employees of GSK Vaccines. The authors TF, MB and RG-S have submitted patent applications for glycoconjugate vaccines against $\mathrm{NmX}$.

\section{REFERENCES}

1. Stephens, D. Biology and pathogenesis of the evolutionarily successful, obligate human bacterium Neisseria meningitidis. Vaccine 27(Suppl 2): B71-B77 (2009).

2. Broderick, M. P., Phillips, C. \& Faix, D. Meningococcal disease in US military personnel before and after adoption of conjugate vaccine. Emerg. Infect. Dis. 21, 377-379 (2015).

3. Hill, D. J., Griffiths, N. J., Borodina, E. \& Virji, M. Cellular and molecular biology of Neisseria meningitidis colonization and invasive disease. Clin. Sci. (Lond) 118, 547-564 (2010).

4. Kratz, M. M. et al. Community-Based Outbreak of Neisseria meningitidis Serogroup C Infection in Men who Have Sex with Men, New York City, New York, USA, 20102013. Emerg. Infect. Dis. 21, 1379-1386 (2015).

5. Kupferschmidt, K. Infectious diseases. Bacterial meningitis finds new niche in gay communities. Science 341, 328 (2013).

6. Caesar, N. M., Myers, K. A. \& Fan, X. Neisseria meningitidis serogroup B vaccine development. Microb. Pathog. 57, 33-40 (2013).

7. Cartwright, K. A. \& Ala'Aldeen, D. A. Neisseria meningitidis: clinical aspects. J. Infect. 34, 15-19 (1997).

8. Tan, L. K., Carlone, G. M. \& Borrow, R. Advances in the development of vaccines against Neisseria meningitidis. N. Engl. J. Med. 362, 1511-1520 (2010).

9. Nadel, S. Prospects for eradication of meningococcal disease. Arch. Dis. Child 97, 993-998 (2012).

10. Costantino, P., Rappuoli, R. \& Berti, F. The design of semi-synthetic and synthetic glycoconjugate vaccines. Expert Opin. Drug Discov 6, 1045-1066 (2011).

11. Tiffay, K., Jodar, L., Kieny, M. P., Socquet, M. \& Laforce, F. M. The Evolution of the Meningitis Vaccine Project. Clin. Infect. Dis. 61(Suppl 5): S396-S403 (2015).

12. Laforce, F. M., Konde, K., Viviani, S. \& Preziosi, M. P. The Meningitis Vaccine Project. Vaccine 25(Suppl 1): A97-A100 (2007).

13. Tzeng, Y. L., Thomas, J. \& Stephens, D. S. Regulation of capsule in Neisseria meningitidis. Crit Rev. Microbiol. 42, 759-772 (2015).

14. Lo, H., Tang, C. M. \& Exley, R. M. Mechanisms of avoidance of host immunity by Neisseria meningitidis and its effect on vaccine development. Lancet Infect. Dis. 9, 418-427 (2009).

15. Eckhardt, M. et al. Molecular characterization of eukaryotic polysialyltransferase-1. Nature 373, 715-718 (1995).

16. Pace, D. Glycoconjugate vaccines. Expert Opin. Biol. Ther. 13, 11-33 (2013).

17. Xie, O., Pollard, A. J., Mueller, J. E. \& Norheim, G. Emergence of serogroup X meningococcal disease in Africa: need for a vaccine. Vaccine 31, 2852-2861 (2013).

18. Maurice, J. Vaccine shortage threatens spread of meningitis in Niger. Lancet $\mathbf{3 8 5}$, 2241 (2015)

19. Fiebig, T. et al. Functional expression of the capsule polymerase of Neisseria meningitidis serogroup X: a new perspective for vaccine development. Glycobiology 24, 150-158 (2014).

20. Fiebig, T. et al. Molecular cloning and functional characterization of components of the capsule biosynthesis complex of Neisseria meningitidis serogroup A: toward in vitro vaccine production. J. Biol. Chem. 289, 19395-19407 (2014).

21. Micoli, F. et al. Development of a glycoconjugate vaccine to prevent meningitis in Africa caused by meningococcal serogroup X. Proc. Natl Acad. Sci. USA 110, 19077-19082 (2013).

22. Berti, F. et al. Relative stability of meningococcal serogroup $A$ and $X$ polysaccharides. Vaccine 30, 6409-6415 (2012).

23. Bröker, M., Costantino, P., DeTora, L., McIntosh, E. D. \& Rappuoli, R. Biochemical and biological characteristics of cross-reacting material 197 CRM197, a non-toxic mutant of diphtheria toxin: use as a conjugation protein in vaccines and other potential clinical applications. Biologicals 39, 195-204 (2011).

24. Micoli, F. et al. Meningococcal $X$ polysaccharide quantification by highperformance anion-exchange chromatography using synthetic N-acetylglucosamine-4-phosphate as standard. Anal. Biochem. 442, 259-261 (2013).

25. Boisier, P. et al. Meningococcal meningitis: unprecedented incidence of serogroup X-related cases in 2006 in Niger. Clin. Infect. Dis. 44, 657-663 (2007).

26. Chen, $C$. et al. A first meningococcal meningitis case caused by serogroup $X$ Neisseria meningitidis strains in China. Chin. Med. J. (Engl. ) 121, 664-666 (2008).

27. Fazio, C. et al. Neisseria meningitidis serogroup X sequence type 2888 , Italy. Emerg. Infect. Dis. 16, 359-360 (2010).

28. Vicente, D., Esnal, O. \& Perez-Trallero, E. Fatal Neisseria meningitidis serogroup X sepsis in immunocompromised patients in Spain. Virulence of clinical isolates. J. Infect. 64, 184-187 (2012).

29. Kilic, A. et al. Neisseria meningitidis serogroup $X$ sequence type 767 in Turkey. J. Clin. Microbiol. 48, 4340-4341 (2010).

30. Delrieu, I. et al. Emergence of epidemic Neisseria meningitidis serogroup $X$ meningitis in Togo and Burkina Faso. PLoS One 6, e19513 (2011).

31. Mutonga, D. M. et al. Epidemiology and risk factors for serogroup $X$ meningococcal meningitis during an outbreak in western Kenya, 2005-2006. Am. J. Trop. Med. Hyg. 80, 619-624 (2009). 
32. MacLennan, C. A. \& Saul, A. Vaccines against poverty. Proc. Natl Acad. Sci. USA 111, 12307-12312 (2014).

33. Shen, A. K. et al. Country ownership and Gavi transition: comprehensive approaches to supporting new vaccine introduction. Health Aff. (Millwood) 35, 272-276 (2016)

34. Berkley, S. Make vaccine coverage a key UN health indicator. Nature 526, 165 (2015).

35. Jones, C. Glycoconjugate vaccines: the regulatory framework. Methods Mol. Biol. 1331, 229-251 (2015).

36. Chilukuri, S. R. et al. Process development and immunogenicity studies on a serogroup ' $X$ ' Meningococcal polysaccharide conjugate vaccine. Biologicals 42, 160-168 (2014).

37. Robinson, J. A. \& Apicella, M. A. Isolation and characterization of Neisseria meningitidis Groups $\mathrm{A}, \mathrm{C}, \mathrm{X}$ and $\mathrm{Y}$ polysaccharide antigens. Infect. Immun. 1, 8-14 (1970).

38. Bundle, D. R., Jennings, H. J. \& Kenny, C. P. Studies on the group-specific polysaccharide of Neisseria meningitidis serogroup $\mathrm{X}$ and an improved procedure for its isolation. J. Biol. Chem. 249, 4797-4801 (1974).

39. Xie, O. et al. Characterization of size, structure and purity of serogroup X Neisseria meningitidis polysaccharide, and development of an assay for quantification of human antibodies. Vaccine 30, 5812-5823 (2012).

40. Keys, T. G., Berger, M. \& Gerardy-Schahn, R. A high-throughput screen for polysialyltransferase activity. Anal. Biochem. 427, 60-68 (2012).
41. Keys, T. G. et al. Engineering the product profile of a polysialyltransferase. Nat Chem. Biol. 10, 437-442 (2014).

42. Romanow, A. et al. Biochemical and biophysical characterization of the sialyl-/ hexosyltransferase synthesizing the meningococcal serogroup W135 heteropolysaccharide capsule. J. Biol. Chem. 288, 11718-11730 (2013).

43. Romanow, A. et al. Dissection of hexosyl- and sialyltransferase domains in the bifunctional capsule polymerases from Neisseria meningitidis $W$ and $Y$ defines a new sialyltransferase family. J. Biol. Chem. 289, 33945-33957 (2014).

44. Litschko, C. et al. The capsule polymerase CsIB of Neisseria meningitidis serogroup L catalyzes the synthesis of a complex trimeric repeating unit comprising glycosidic and phosphodiester linkages. J. Biol. Chem. 290, 24355-24366 (2015).

\section{(c) (i)}

This work is licensed under a Creative Commons Attribution 4.0 International License. The images or other third party material in this article are included in the article's Creative Commons license, unless indicated otherwise in the credit line; if the material is not included under the Creative Commons license, users will need to obtain permission from the license holder to reproduce the material. To view a copy of this license, visit http://creativecommons.org/licenses/ by/4.0/

(c) The Author(s) 2016

Supplementary Information accompanies the paper on the npj Vaccines website (http://www.nature.com/npjvaccines) 